\title{
Cetamina: aspectos gerais e relação com a esquizofrenia
}

Ketamine: General Aspects and Relationship with Schizophrenia

\author{
Silvânia M. M. VASCONCELOS ${ }^{1}$ \\ Marcelo de Moraes Andrade ${ }^{1}$ \\ Paula Matias Soares ${ }^{1}$ \\ Breitner Gomes Chaves ${ }^{1}$ \\ Manoel C. A. Patrocínio ${ }^{1}$ \\ Francisca Cléa Florenç̧a Sousa ${ }^{2}$ \\ DANIELle SilveIra MaCÊDO ${ }^{3}$
}

\begin{abstract}
Resumo
A cetamina é uma droga anestésica desenvolvida em 1965 pelos laboratórios norte-americanos Parke \& Davis, tendo como objetivo principal sua utilização em anestesias humanas e veterinárias. Entretanto, seu uso tornou-se constante entre os jovens, sendo consumida em festas como um potente alucinógeno. Já quanto a pesquisas laboratoriais, essa droga tem sido utilizada como modelo para induzir esquizofrenia em animais. Com o objetivo de realizar-se um estudo de revisão da cetamina como anestésico e potencial modelo de esquizofrenia, foi feita uma pesquisa bibliográfica na internet, utilizando programas de pesquisa científica ( $P u b m e d$, Medline e Lilacs), além de pesquisa em trabalhos relacionados ao assunto. A administração da cetamina no homem promove o bloqueio dos receptores glutamatérgicos ionotrópicos do tipo N-metil-D-aspartato (NMDA) e antagoniza os receptores de acetilcolina nicotínicos e muscarínicos, bem como os receptores monoaminérgicos e opióides. O bloqueio dos receptores glutamatérgicos promoverá um quadro sintomático semelhante ao de um paciente esquizofrênico. Além disso, a administração da cetamina durante a sinaptogênese pode lesar neurônios corticais, límbicos, talâmicos e estriatais, promovendo uma disfunção na neurotransmissão glutamatérgica e propiciando a manifestação de sintomas psicóticos na vida adulta. Entre esses sintomas, podemos citar o surgimento da esquizofrenia. Somando-se a isso, a droga proporciona uma série de efeitos sistêmicos, desde uma simples anestesia, passando pela sedação, depressão respiratória e até a morte.
\end{abstract}

Palavras-chave: Cetamina, efeitos sistêmicos, esquizofrenia, modelo animal.

\section{Abstract}

Ketamine is an anesthetic agent developed in 1965 by Park \& Davis laboratories to be used as a general anesthetic in humans and animals. However, its use became popular among young people and it's frequently available at parties to produce hallucination. In laboratorial researches, this drug has been used as a model of schizophrenia in animals. This work intend to realize a review

Recebido: 19/04/2004 - Aceito: 28/01/2005

1 Departamento de Ciências Fisiológicas, Universidade Estadual do Ceará (UECE)

2 Departamento de Fisiologia e Farmacologia, Universidade Federal do Ceará (UFC)

3 Curso de Fisioterapia da Faculdade Integrada do Ceará (FIC)

Endereço para correspondência: Dra. Silvânia Vasconcelos. Universidade Estadual do Ceará. Av. Soriano Albuquerque, 185 - Apto. 1401, 60130-160, Fortaleza - CE. Fone: (85) 299-2724, e-mail: claussil@bol.com.br / silvania_vasconcelos@yahoo.com.br

Este estudo recebeu auxílio da CAPES e da FAPESP (processos 03/12337-8 e 02/11247-2). 
article about ketamine as an anesthetic and potential schizophrenia model, through a bibliographic research in sites of scientific research, as Pubmed, Medline, and Lilacs and some papers related to this subject. Ketamine administration in humans produces a blockage at glutamate N-methyl-Daspartate (NMDA) receptors, antagonizes nicotinic and muscarinic acetylcholine receptors, as well as opioids and monoaminergic systems. The blockage of glutamatergic receptors induces symptoms very similar to the ones presented by schizophrenic patients. Besides this, ketamine administration during synaptogenesis can injury cortical, limbic, thalamic and striatal neurons, producing a dysfunction in glutamatergic neurotransmission, leading to manifestation of psychotic symptoms during adult life. Among these symptoms, we can mention the development of schizophrenia. The drug also produces systemic effects that go from a simple anesthesia, sedation, respiratory depression until to death.

Keywords: Ketamine, systemic effects, schizophrenia, animal model.

\section{Introdução}

A cetamina é um derivado do cloridrato de fenciclidina (phencyclidine hydrochloride-PCP) (Micallef et al., 2003), sintetizado por Stevens em 1965 e tendo como principal uso a anestesia em humanos e animais. Essa droga foi introduzida no mercado pelo laboratório americano Parke \& Davis e na clínica a partir da década de 1960 (Domino et al., 1965). É referida na literatura como "anestésico dissociativo", devido a uma perda sensorial marcante e analgesia, assim como amnésia e paralisia do movimento, sem perda real da consciência (Rang et al., 2004; Morgan et al., 2004), ocorrendo intensa sensação de dissociação do meio. A cetamina é usada no trauma e em procedimentos cirúrgicos de emergência, bem como na medicina veterinária (Wagner et al., 2002). A principal desvantagem da droga é a ocorrência de delírios e comportamentos irracionais durante a recuperação, por isso seu uso é indicado em conjunção com um benzodiazepínico (Barash et al., 1991).

Kim et al. (1980), em um estudo sobre a esquizofrenia, sugeriram que pessoas possuidoras desse tipo de patologia apresentavam uma diminuição na concentração de glutamato no líquido cefalorraquidiano. Contudo, apesar de inconsistentemente reproduzido, esse achado originou a teoria glutamatérgica da esquizofrenia e impulsionou uma série de estudos na área (Bressan e Pilowskv, 2003).

Trabalhos realizados recentemente têm demonstrado o uso da cetamina como indutora de sintomas positivos e negativos similares àqueles associados com a esquizofrenia (Keilhoff et al., 2004; Pietraszek, 2003) tanto em humanos (Keilhoff et al., 2004; Bressan e Pilowskv, 2003) quanto em animais, sendo proposto como um possível modelo de esquizofrenia (Becker et al., 2003; Keilhoff et al., 2004). Nesse sentido, sua principal ação estaria relacionada a uma hipofunção glutamatérgica indutora dos efeitos comportamentais da doença.

Esquizofrenia é um transtorno psiquiátrico devastador que acomete aproximadamente $1 \%$ da população ao longo da vida (Bressan e Pilowskv, 2003), uma complexa desordem caracterizada por sintomas positivos (delírios e alucinações) e negativos (embotamento afetivo, retraimento emocional e pensamento estereotipado). Embora essa patologia seja bem descrita na literatura, pouco se conhece sobre seus mecanismos neuro e psicopatológicos exatos (Becker et al., 2003; Bressan e Pilowskv, 2003).

Com o propósito de estudar o modelo glutamatérgico efetivo de indução da esquizofrenia em animais, foi feita esta revisão bibliográfica pela internet, na qual textos relacionados ao tema forma levantados. Os programas de busca utilizados foram os do PubMed, Medline Lilacs (palavras-chave: ketamine and schizophrenia), bem como textos envolvendo dados epidemiológicos, farmacocinética, farmacodinâmica, toxicidade, além de pesquisas laboratoriais de associação do glutamato e da esquizofrenia.

\section{Cetamina como anestésico sistêmico}

Estudos animais indicam que receptores glutamatérgicos do tipo N-metil-D-aspartato (NMDA) presentes no corno dorsal da medula têm um importante papel no mecanismo de sensibilização central. Antagonistas do receptor NMDA freiam a hiperatividade de neurônios do corno dorsal posterior à ativação prolongada de neurônios aferentes primários e inibem 
o comportamento nociceptivo induzido por lesões nos tecidos periféricos ou nervos (Coderre et al., 1993; Dickenson, 1997).

A cetamina é um potente antagonista nãocompetitivo do receptor NMDA (Haas e Harper, 1992) e atua no sítio de ligação do PCP, presente neste canal iônico. $\mathrm{O}$ termo não-competitivo significa que essa droga, além da PCP e da dizocilpina (MK801), não compete pelo mesmo sítio de ligação que o glutamato (Bressan e Pilowskv, 2003). Ambos os isômeros da cetamina têm afinidade por esse receptor. A inibição da dor por essa droga ocorre em concentrações plasmáticas abaixo de $1 \mathrm{mM} / \mathrm{L}$, existindo uma correlação altamente significativa entre os níveis plasmáticos da droga e o alívio da dor (Eide et al., 1995).

Estudos clínicos randomizados mostraram que a cetamina inibe a dor aguda causada por vários estímulos nocivos, incluindo isquemia, estímulos químicos (Park et al., 1995), elétricos e calor (Ilkjaer et al., 1996), além de ser preferencialmente utilizada em procedimentos cirúrgicos associados à dor intensa e/ou à elevada ansiedade (Marco et al., 2000). O bloqueio do receptor NMDA induzido pela cetamina também inibe a somação temporal de estímulos elétricos e mecânicos repetidos (Arendt-Nielsen et al., 1995). Portanto, evidências clínicas sugerem que a cetamina inibe a sensibilização central (Camu e Vanlersbergh, 2002). Além disso, estudos controlados também demonstraram que tal droga inibe de forma significativa a dor espontânea de pacientes com diferentes tipos de dor crônica, incluindo dor neuropática central e periférica, fibromialgia e dor isquêmica crônica (Persson et al., 1998). Essa droga também reduz alodinia em pacientes com dor neuropática (Eide et al., 1995).

\section{Cetamina como droga de abuso}

O uso de drogas psicotrópicas é universal, sendo comum às mais diferentes culturas humanas. De acordo com o efeito provocado no sistema nervoso central, essas substâncias podem ser classificadas como estimulantes, depressoras ou perturbadoras de seu funcionamento (alucinógenos) (Almeida e Silva, 2000). A cetamina, freqüentemente disponível em festas (Bascuñana et al., 2003), encontra-se com uma classificação duvidosa, pois sua apresentação como droga de abuso geralmente é associada à 3-4 metilenodioximetanfetamina (MDMA ou, como é popularmente conhecida, ecstasy) (Wolff et al., 1995). No entanto, Cruz e López (2001) classificam-nas como anestésicos dissociativos, demonstrando uma divisão mais complexa desses fármacos.

Bascuñana et al. (2003) colocam-na como uma das principais drogas consumidas em megafestas, juntamente com o ácido gama-hidroxibutírico (GHB), anfetaminas, ecstasy, cocaína, ácido lisér- gico (LSD) e derivados de cannabis ou canabinóides (maconha e haxixe).

A forma de consumo mais comum é a inalatória (Bascuñana et al., 2003); no entanto, a cetamina pode ainda se apresentar sob a forma líquida ou em comprimido, sendo injetada, deglutida ou fumada juntamente com maconha ou tabaco. Comumente, essa droga recebe nomes como "K", "Special K", "Vitamina K", "Pó dos Anjos" etc. (Cruz e López, 2001; Bascuñana et al., 2003), tendo meia-vida de aproximadamente duas horas (Domino et al., 1984).

É um fármaco que atua sobre o sistema límbico e córtex cerebral, aumentando a excitação elétrica dessas regiões, além dos gânglios da base e hipocampo, causando elevação do tônus simpático (Bascuñana et al., 2003). O mecanismo de ação da droga, como já descrito anteriormente, envolve a inibição do receptor NMDA, importante na neuroquímica do comportamento e informação sensorial (Cruz e López, 2001).

Muitos dos efeitos psicomiméticos dessa droga são transitórios, reversíveis e influenciados pelas condições de tempo, dose e administração (Keilhoff et al., 2004). Doses baixas de cetamina, utilizadas pelos viciados, produzem melhora no humor e sintomas psiquiátricos como perda da realidade, despersonalização, alucinações visuais, sonhos prazerosos ou não (Zacny e Galinkin, 1999), perda de atenção, de habilidade de aprendizado e de memória. Doses mais altas causam vômitos, fala arrastada, amnésia, redução da função motora, delírio com ou sem agitação psicomotora, movimentos tônicos-clônicos, taquicardia ou bradicardia, hipotensão e depressão respiratória (Bascuñana et al., 2003; Mathias et al., 1999). Usuários de altas doses reportam experiências como saída do próprio corpo ou proximidade à morte, como também distúrbios visuais ou flashbacks que podem durar dias ou semanas após a exposição. Os possíveis efeitos sobre a cognição ou psiquiátricos, produzidos pelo uso da droga em longo prazo, não foram suficientemente estudados em usuários (Freeze et al., 2002). Tolerância e dependência foram reportadas (Dotson et al., 1995), mas estudos adicionais são necessários para se determinarem os sintomas da retirada e um tratamento eficaz para ela.

\section{Cetamina e esquizofrenia}

A esquizofrenia é uma doença complexa caracterizada por sintomas positivos ou produtivos, sintomas negativos ou deficitários (Kaplan et al., 2003) e disfunção cognitiva (perda de memória de curta duração, déficits de atenção etc.) (Corbett et al., 1995; Morgan et al., 2004).

Diferentes abordagens científicas (modelos animais, estudos neuroendocrinológicos, post-mortem, psicofarmacológicos, genéticos e de neuroimagem) têm sido empregadas para investigar a esquizofrenia 
(Bressan e Pilowskv, 2003). Contudo, como essa doença apresenta-se sem uma patofisiologia ou etiologia conhecidas, modelos animais tornam-se válidos para seu estudo, embora tais modelos não possam expressar alguns sintomas característicos (Becker et al., 2003).

Segundo Bressan e Pilowskv (2003), o sistema dopaminérgico é o mais profundamente estudado na esquizofrenia, levantando a explicação mais aceita da hipótese de hiperfunção dopaminérgica. As evidências para esse fato encontram-se na indução de quadros psicóticos por anfetamina (indutor de liberação de dopamina) e no efeito terapêutico de neurolépticos pelo bloqueio de receptores dopaminérgicos tipo $\mathrm{D}_{2}$ (Kapur e Seeman, 2001; Pietraszek, 2003). Contudo, essas drogas atuam principalmente na melhoras dos sintomas positivos da doença.

Estudos sugerem que os sintomas psicóticos resultariam de um desequilíbrio entre os sistemas dopaminérgicos cortical e subcortical. $\mathrm{O}$ hipofuncionamento do sistema dopaminérgico do córtex pré-frontal pode ser o responsável pelo desenvolvimento de sintomas negativos, enquanto os sintomas positivos poderiam ser atribuídos ao aumento da atividade dopaminérgica no sistema límbico (Kahn e Davis, 1995; Knable e Weinberger, 1997). Esses sistemas (cortical e subcortical), segundo Pietraszek (2003), estão funcionalmente relacionados via sistema glutamatérgico, sugerindo que sua disfunção poderia também precipitar sintomas psicóticos.

Estudos têm enfatizado a participação de vários sistemas de neurotransmissores na fisiopatologia da esquizofrenia (Becker et al., 2003; Bernstein et al., 2003). Entre esses sistemas, o glutamatérgico tem recebido uma atenção especial, principalmente pela sua inervação no córtex cerebral.

Olney (1988) descreveu a hipótese glutamatérgica para a esquizofrenia, estabelecendo uma relação entre o bloqueio da função dos receptores NMDA por fenciclidina e os sintomas psicóticos "tipo" esquizofrenia. Assim, a hipofunção do receptor NMDA por si só pode ser um possível mecanismo para explicar a esquizofrenia (Olney, 1988; Olney et al., 1995).

Segundo Grotta et al. (1995), as ações psicomiméticas de vários antagonistas do receptor NMDA podem estar relacionadas ao bloqueio neste receptor. A fenciclidina e a cetamina, antagonistas nãocompetitivos do complexo receptor de NMDA, exacerbaram sintomas psicóticos em pacientes esquizofrênicos e induziram sintomas positivos e negativos semelhantes a essa doença em indivíduos saudáveis (Adler et al., 1999; Luby et al., 1959). Também Krystal et al. (1994) demonstraram que a administração de cetamina $(0,1$ ou $0,5 \mathrm{mg} / \mathrm{kg})$ em indivíduos saudáveis produziu comportamento semelhante aos sintomas positivos e negativos da esquizofrenia, bem como alterações da percepção, prejuízo na fluência verbal e sintomas similares ao estado dissociativo.
Entre os antagonistas de NMDA, a cetamina tem recebido atenção em uma série de trabalhos na literatura científica (Miyamoto et al., 2001; Becker et al., 2003; Duncan et al., 2000). O interesse pelo seu estudo surgiu a partir de um trabalho realizado por Lahti et al. (1995), no qual a cetamina de forma doseresposta induziu sintomatologia psicótica em voluntários humanos sadios. Esse estudo também foi realizado em voluntários esquizofrênicos. Nessa pesquisa, cada voluntário participou de quatro sessões nas quais recebia uma das três doses de cetamina $(0,1,0,3$ ou $0,5 \mathrm{mg} / \mathrm{kg}$ ) ou placebo em um estudo duplo-cego. A droga produziu sintomas psicóticos dose-dependente em voluntários humanos sadios, além de causar um aumento de mesma magnitude nos voluntários esquizofrênicos. Os indivíduos sadios tiveram sintomas positivos e negativos e aqueles que apresentavam a patologia só demonstraram um aumento nos sintomas positivos. Voluntários normais experimentaram delírios e distorções visuais e auditivas, enquanto os esquizofrênicos reportaram fenômenos semelhantes somados às alucinações. Todos os grupos apresentaram desordens nos pensamentos, e esses sintomas duraram de 20 a 40 minutos.

Estudos usando tomografia por emissão de pósitrons (PET) demonstraram que doses subanestésicas de cetamina produzem aumentos bilaterais nas atividades metabólicas dos córtices pré-frontal e cingulado anterior em humanos (Breier et al., 1997; Langsjo et al., 2003; Langsjo et al., 2004). A existência de uma inter-relação anatômica e funcional entre os sistemas dopaminérgicos e glutamatérgicos no sistema nervoso central sugere que a inibição de receptores NMDA pode influenciar a neurotransmissão dopaminérgica (Vasiliadis et al., 1999, Zheng et al., 1999). Por exemplo, antagonistas do receptor NMDA aumentam o disparo de neurônios dopaminérgicos (Murase et al., 1993) e causam liberação de dopamina neuroanatomicamente seletiva em roedores (Adams e Moghaddam, 1998; Rao et al., 1989). Em humanos, estudos de PET medindo a ocupação dos receptores dopaminérgicos após a administração de cetamina sugeriram que antagonistas NMDA aumentam a liberação de dopamina no núcleo estriado (Smith et al., 1998), e esses efeitos são suprimidos pelo menos em parte por drogas antipsicóticas (Corbett et al., 1995; Irifune et al., 1995). A cetamina mostra afinidade muito similar pelos receptores $\mathrm{NMDA}$ e $\mathrm{D}_{2}$, com menor afinidade por sítios $5 \mathrm{HT}_{2}$. Em trabalho recente, Becker et al. (2003) demonstraram que a cetamina administrada por cinco dias causa alterações nas neurotransmissões dopaminérgica, glutamatérgica e serotoninérgica, produzindo aumento do receptor $\mathrm{D}_{2}$ no hipocampo, redução do receptor glutamatérgico no córtex frontal, aumento do transportador da dopamina no estriado e do transportador da serotonina no estriado, hipocampo e córtex frontal. 
Em laboratório de pesquisa está-se utilizando a cetamina como um modelo experimental efetivo para induzir esquizofrenia em animais (roedores), e os resultados preliminares, com cetamina na dose de 30 $\mathrm{mg} / \mathrm{kg}$, intraperitoneal, demonstraram um aumento da atividade locomotora, comportamento rotacional e movimento de descolamento da cabeça de um lado para o outro (direito e esquerdo) (Andrade et al., 2004; Malveira et al., 2004).

\section{Conclusões}

A cetamina é uma droga que produz uma série de efeitos sistêmicos, dentre eles anestesia geral e analgesia, principalmente devido ao bloqueio dos receptores glutamatérgicos-NMDA. Ela causa agitação e dissociação do meio (por isso, sendo usada como droga de abuso), bem como efeitos psicóticos, provavelmente porque o bloqueio de receptores NMDA interfere com a neurotransmissão dopaminérgica, a qual está envolvida com a esquizofrenia. Essas evidências levaram diversos autores a pesquisarem modelos propícios para maiores estudos na área, despertando, assim, a cetamina como um possível modelo de esquizofrenia. Além disso, o direcionamento para estudo em modelos animais mostrou-se importante no sentido de auxiliar no entendimento desta doença e, conseqüentemente, no possível desenvolvimento de drogas terapêuticas antipsicóticas.

\section{Referências bibliográficas}

Adams, B.; Moghaddam, B. - Corticolimbic dopamine neurotransmission is temporally dissociated from the cognitive and locomotor effects of phencyclidine. $J$ Neurosci 18: 5545-54, 1998.

Adler, C.M.; Malhotra, A.K.; Elman, I. et al. - Comparison of ketamine - induced thought disorder in healthy volunteers and thought disorder in schizophrenia. Amer J Psychiat 156: 1646-9, 1999.

Almeida, S.P.; Silva, M.T.A. - Histórico. Efeitos e mecanismo de ação do êxtase (3-4 metilenodioximetanfetamina): revisão da literatura. Ver Panam Salud Publica/Pan Am $J$ Public Health 8: 393-402, 2000.

Andrade, M.M.; Chaves, B.G.; JucÁ, N.B.H. et al. - Blockade of the antidepressant-like effects of ketamine with atypical neuroleptics. ${ }^{\text {st }}$ Neuroscience Symposium. Natal - Brasil, 2004.

Arendt-Nielsen, L.; Petersen-Felix, S.; Fischer, M. et al. - The effect of $\mathrm{N}$-methyl-D-aspartic antagonist (ketamine) on single and repeated nociceptive stimuli: a placebo-controlled experimental humam study. Anesth Analg 85: 63-8, 1995.

Barash, P.G.; Cullen, B.F.; Stoelling, R.K. - Manual de Anestesiologia Clínica. Barueri: Manole, 1991.

Bascuñana, J.J.A.; GutiérRez, R.C.; GonzaléZ, J.L.M. et al. Drogas de diseño: un Nuevo paciente crítico. Manejo de las intoxicaciones por drogas consumidas en macrofiestas. Salud Rural 20: 1-9, 2003.

Becker, A.; Peters, B.; Schroeder, H. et al. - Ketamine-induced changes in art behavior: a possible animal model of schizophrenia. Prog Neuro-psychoph 27: 687-700, 2003.

Bernstein, H.G.; Becker, A.; Kellhoff, G. et al. - Brain regionspecific changes in the expression of calcium sensor proteins after repeated application of ketamine in rats. Neurosci Lett 339: 95-8, 2003.
Breier, A.; Malhotra, A.K.; Pinals, D.A. et al. - Association of ketamine-induced psychosis with focal activation of the prefrontal cortex in healthy volunteers. $A m \mathrm{~J}$ Psychiatry 154: 805-11, 1997.

Bressan, R.A.; PILowskv, L.S. - Hipótese glutamatérgica da esquizofrenia. Rev Bras Psiquiatr 25: 177-83, 2003.

Camu, F.; Vanlersbergh, C. - Pharmacology of systemic analgesics. Best practice \& Research clinical. Anaesthesiology 16: 475-88, 2002.

Coвett, R.; Самасно, F.; Woods, A.T. et al. - Antipsychotic agents antagonize non-competitive $\mathrm{N}$-methyl-D aspartate antagonist-induced behaviors. Psychopharmacol 120: 67-74, 1995.

Coderre, T.J.; Katz, J.; Vaccarino, A.L. et al. - Contribution of central neuroplasticity to pathological pain: review of clinical and experimental evidence. Pain 52: 259-85, 1993.

Cruz, S.L., López, C. - Fármacos que producen dependência física y adicción. Avance y perspectiva/Altar 211-7, 2001.

DiCKenson, A. - Mechanisms of central hypersensitivity: excitatory amino acid mechanisms and their control. In: Dickenson, A.; Besson, J.M. (eds) The pharmacology of Pain. Berlin: Springer Verlag, pp. 167-210. 1997.

Domino, E.F.; Codoff, P.; Conssen, G. - Pharmacologic effects of Cl-581, a new dissociative anesthetic in man. Clin Pharmacol Ther 6: 279, 1965.

Domino, E.F.; Domino, S.E.; Sмiтн, R.E. et al. - Ketamine kinetics in unpremedicatedand diazepam-premedicated subjects. Clin Pharmacol Ther 36: 545-53, 1984.

Dotson, J.W.; AcKerman, D.L.; WeSt, L.J. - Ketamine abuse. J Drug Issues 25: 751-7, 1995.

Duncan, G.E.; Miyamoto, S.; LeIPZIG, J.N. et al. - Comparison of the effects of clozapine, risperidone, and olanzapine 
on ketamine-induced alterations in regional brain metabolism. J Pharmacol Exp Ther 293: 8-14, 2000.

Eide, P.K.; Stubhaug, A.; Oye, I. et al. - Continuous subcutaneous administration of the $\mathrm{N}$-methyl-D-aspartic acid (NMDA) receptor antagononist ketamine in the treatment of postherpetic neuralgia. Pain 61: 221-8, 1995.

Freese, T.E.; Mıотto, K.; Reback, C.J. - The effects and consequences of selected club drugs. J Subst Abuse Treat 23: 151-6, 2002.

GrotTA, J.; ClaRK, W.; CoulL, B. et al. - Safety and tolerability of the glutamate antagonist CGS 19755 (Selfotel) in patients with acute ischemic stroke. Results of a phase Ila randomized trial. Stroke 26: 602-5, 1995.

HaAs, D.A.; HaRPER, D.G. - Ketamine: a review of its pharmacologic properties and use in ambulatory anesthesia. Anesth Prog 39: 61-8, 1992.

IlkJaer, S.; Petersen, K.L.; Brennum, J. et al. - Effect of systemic $\mathrm{N}$-methyl-D-aspartic receptor antagonist (ketamine) on primary and secondary hyperalgesia in humans. $\mathrm{Br} \mathrm{J}$ Anaesth 76: 829-34, 1996.

IrIFUne, M.; Shimizu, T.; Nomoto, M. et al. - Involvement of Nmethyl-D-aspartate (NMDA) receptors in noncompetitive NMDA receptor antagonist-induced hyperlocomotion in mice. Pharmacol Biochem Beh 51: 291-6, 1995.

Kaplan, H.I.; Sadock, B.J.; GrebB, J.A. - Compêndio de Psiquiatria: Ciências do Comportamento e Psiquiatria Clínica. $7^{\mathrm{a}}$ ed., Porto Alegre: ArtMed, 2003.

Kapur, S.; Seeman, P. - Does fast dissociation from the dopamine $D_{2}$ receptor explain the action of atypical antipsychotics? A new hypotesis. Am J Psychiatry 158: 360-9, 2001.

KaHN, R.S.; Davis, K.L. - New developments in dopamine and schizophrenia. In: Bloom, F.E.; Kupfer, D.J. Psychopharmacology: The Fourth Generation of Progress. New York: Raven Press. pp. 1193-201, 1995.

KeILhoff, G.; BeCKer, A.; Grecksch, G. et al. - Repeated application of ketamine to rats induces changes in the hippocampal expression of parvalbumin, neuronal nitric oxide synthase and cFOS similar to those found in human schizophrenia. Neuroscience 126: 591-8, 2004.

KIm, J.S.; Kornhuber, H.H.; Schmid-Burgk, W. et al. - Low cerebrospinal fluid glutamate in schizophrenic patients and a new hypothesis on schizophrenia. Neurosc Lett 20: 379-82, 1980.

KNABLE, M.B.; WeinBerGeR, D.R. - Dopamine, the pre-frontal cortex and schizophrenia. J Psychopharmacol 11: 123-236, 1997.

Krystal, J.H.; KarPer, L.P.; SelbyL, J.P. et al. - Subanesthetic effects of the noncompetitive NMDA antagonist, ketamine, in humans. Psychotomimetic, perceptual, cognitive, and neuroendocrine responses. Arch Gen Psychiatry 51: 199-214, 1994.

Lahti, A.C.; Holcomb, H.H.; Weller, M.A. et al. - Effects of ketamine on behavior and $\mathrm{rCBF}$ in schizophrenia and normal individuals. Biol Psychiatry 41: 165-9, 1997.

Langsjo, J.W.; Kaisti, K.K.; Aalto, S. et al. - Effects of subanesthetic doses of ketamine on regional cerebral blood flow, oxygen consumption, and blood volume in humans. Anesthesiology 99: 614-23, 2003.

Langsjo, J.W.; Salmi, E.; Kaisti, K.K. et al. - Effects of subanesthetic ketamine on regional cerebral glucose metabolism in humans. Anesthesiology 100: 1065-71, 2004.

Lahti, A.C.; Koffel, B.; LaPorte, D. et al. - Subanesthetic doses of ketamine stimulate psychosis in schizophrenia. Neuropsychopharmacology 13: 9-19, 1995.

LuBY, E.D.; CoHen, B.D.; Rosenbaum, G. et al. - Study of a new schizophrenomimetic drug: serenyl. Arch Neurol Psychiatry 71: 363-9, 1959.

Malveira, G.C.H.; Sousa, F.B.M.; Vasconcelos, S.M.M. et al. Effect of typical and atypical neuroleptics on ketamineinduced behavioral alterations in open field test. $1^{\text {st }}$ Neuroscience Symposium. Natal-Brasil, 2004.

Marco, J.P.; Serigó, L.G.; Sorolla, J.M.F. et al. - Analgesia y sedación en urgencies. Emergências 12: 272-9, 2000.

Mathias, L.A.S.T.; Freitas, P.L.A.; Mercado, C.R. et al. - Efeitos colaterais centrais da $S(+)$ cetamina em pacientes submetidas a curetagem uterina. Rev Bras Anestesiol 49: 15A, 1999.

Micallef, J.; Tardieu, S.; Gentile, S. et al. - Évaluation psychocomportementale de l'administration de faible dose de kétamine chez le sujet sain. Neurophysiologie Clinique 33: 138-47, 2003.

Miramoto, S.; Mallman, R.B.; Lieberman, J.A. et al. - Blunted brain metabolic response to ketamine in mice lacking $D(1 A)$ dopamine receptors. Brain Res 894: 167-80, 2001.

Morgan, C.J.A.; Mofeez, A.; Brandner, B. et al. - Acute effects of ketamine on memory systems and psycotic symptoms in healthy volunteers. Neuropsycopharmacology 29: 208-18, 2004.

Murase, S.; Mathe, J.M.; Grenhoff, J. et al. - Effects of dizocilpine (MK-801) on rat midbrain dopamine cell activity: differential action on firing pattern related to anatomical localization. J Neural Transm 91: 13-25, 1993.

OLNEY, J.W. - Endogenous excitotoxins and neuropathological disorders. In: Lodge D (ed.). Excitatory Amino acids in health and disease. England: John Wiley \& Sons, pp 337-51, 1988.

Olney, J.W.; FARBER, N.B. - NMDA antagonists as neurotherapeutic drugs, psychotogens, neurotoxins, and research tools for studying schizophrenia. Neuropsychopharmacology 13: 335-45, 1995.

Park, K.M.; Max, M.B.; Rabinovitz, E. et al. - Effects of intravenous ketamine, alfentanil or placebo on pain pinprick by hyperalgesia and allodinia produced by intradermal capsaicin in human subjects. Pain 63: 16372, 1995.

Persson, J.; Hasselstrom, L.; WikLund, B. et al. - The analgesic effect of recemic ketamine in patients with chronic ischemic pain due to lower extremity arteriosclerosis obliterans. Acta Anaesthesiol Scand 42: 750-8, 1998.

Pietraszek, M. - Significance of dysfunctional glutamatergic transmission for the development of psychotic symptoms. Pol J Pharmacol 55: 133-54, 2003. 
Rang H.P.; Dale M.M.; RitTer J.M. - Farmacologia. $5^{\mathrm{a}}$ ed. Rio de Janeiro: Elsevier, 2004.

RAO, T.S.; KIM, H.S.; LeHMAN, J. et al. - Differential effects of phencyclidine (PCP) and ketamine on mesocortical and mesostriatal dopamine release in vivo. Life Sci 45: 106772, 1989.

Smith, G.S.; Schloesser, R.; Brodie, J.D. et al. - Glutamate modulation of dopamine measured in vivo with positron emission tomography (PET) and C-11 raclopride in normal human subjects. Neuropsychopharmacol 18: 18-25, 1998.

VASILIADIS, H.; ELEE, R.; DEWAR, K.M. - Interaction between dopamine and glutamate receptors following treatment with NMDA receptor antagonists. Eur J Pharmacol 386: 155-63, 1999.
Wagner, A.E.; Walton, J.A.; Hellyer, P.W. et al. - Use of low doses of ketamine administered by constant rate infusion as an adjunct for postoperative analgesia in dogs. J Am Vet Med Assoc 221: 72-5, 2002.

WolfF, K.; HaY, A.W.; SHerlock, K. et al. - Contents of "ecstasy" [letter]. Lancet 346: 1100-1, 1995.

ZacnY, J.P.; Galinkin, J.L. - Psychotropic drugs used in anesthesia. Practice, abuse, liability and epidemiology of abuse. Anesthesiology 90: 269-88, 1999.

Zheng, P.; ZhANG, X.X.; Bunney, B.S. et al. - Opposite modulation of cortical N-methyl-D-aspartate receptor-mediated responses by low and high concentrations of dopamine. Neuroscience 91: 527-35, 1999. 